\title{
DESIGN OF DIGITAL VIDEO WATERMARKING SCHEME USING MATLAB SIMULINK
}

\author{
Prachi V. Powar ${ }^{1}$, S.S.Agrawal ${ }^{2}$ \\ ${ }^{1} P G$ Student, ${ }^{2}$ Asst.Professor, E \& TC Department, SKNCON, Pune, India \\ prachipowar1@email.com, sujata.agarwal@gmail.com
}

\begin{abstract}
Due to increase in growth of internet users of networks are increasing rapidly. Owners of the digital products are concerned about illegal copying of their products. Security and copyright protection are becoming important issues in multimedia applications and services. Digital watermarking is a technology used for copyright protection of digital media. Here ownership information data called watermark is embedded into the digital media without affecting its perceptual quality. In case of any dispute, the watermark data can be detected or extracted from the media and use as a proof of ownership. Digital video watermarking scheme based on Discrete Wavelet Transform is addressed in this paper. Design of this scheme using Matlab Simulink is proposed. Embedded watermark is robust against various attacks that can be carried out on the watermarked video.
\end{abstract}

Index Terms:Digital video, Discrete Wavelet Transform, Binary watermark.

\section{INTRODUCTION}

The use of digital multimedia content is increased large amount of data is transfer and distributed easily. This development will benefit multimedia product owners as sales will increase. Also it will pose challenge to their ownership as most of multimedia products are distributed in insecure format. These products can be transmitted and redistributed easily without any authentication as various tools are available at no cost. So there is need for copyright protection of multimedia data. Video become an important tool for the entertainment and educational industry[2].Digital video watermarking is new technology used for copyright protection of digital media.[1]It inserts authentication information in multimedia data which can be used as proof of ownership.

The watermarking technique is used for data hiding. Video watermarking algorithms normally prefers robustness. Most of the proposed video watermarking schemes are based on the techniques of image watermarking. But video watermarking introduces some issues not present in image watermarking. [2] Watermarking techniques can be classified into spatial or frequency domain by place of application. Spatial domain watermarking is performed by modifying values of pixel color samples of a video frame whereas watermarks of frequency domain techniques are applied to coefficients obtained as the result of a frequency transform of either a whole frame or single block-shaped regions of a frame[6].
Here we propose an implementation of robust video watermarking scheme using Discrete Wavelet Transform Matlab Simulink is used for software design. It is a simulation tool for dynamic system modelling, simulation and comprehensive analysis. It can call MATLAB dominant function library it save a lot of code writing work, and the user can immediately see the results of simulation. [7].

\subsection{Video Watermarking}

Maximum occurrences of copyright violation and distribution happen for video media content. So Video Watermarking is one of the most accepted techniques among the various Watermarking techniques currently in use.

\subsection{Requirements for video water marking}

Requirements for video Watermarking are as follows:

1. Video data is subject to increased attacks than any othermedia.

2. Video content is sensitive to distortions and Watermarking may degrade the quality.

3. Video compression algorithms are computationally rigorous.

4. Video require large bandwidth that is why it is mostlycarried in compressed domain. So Watermarkingalgorithmis also adaptable for compress area processing.

Most commonly used transforms are

1.Discrete Fourier Transform (DFT),

2.Discrete Cosine Transform (DCT),

3. Discrete Wavelet Transform (DWT). 


\section{SCHEME OF IMPLEMENTATION}

\subsection{Discrete Wavelet Transform (DWT)}

The Discrete Wavelet Transform (DWT) is used in a wide variety of signal processing applications. 2-D discrete wavelet transform (DWT) decomposes an image or a video frame into sub-images, 3 details and 1 approximation. The 2-D DWT is an application of the 1-D DWT in both the horizontal and the vertical directions. DWT separates the frequency band of an image into a lower resolution approximation sub-band (LL) as well as horizontal (HL), vertical (LH) and diagonal (HH) detail components.

Watermark is embedded in low frequencies obtained by Wavelet decomposition which increases the robustness. So that resultant watermark video become susceptible to different attacks that have low pass characteristics like filtering, lossy compression and geometric distortions.
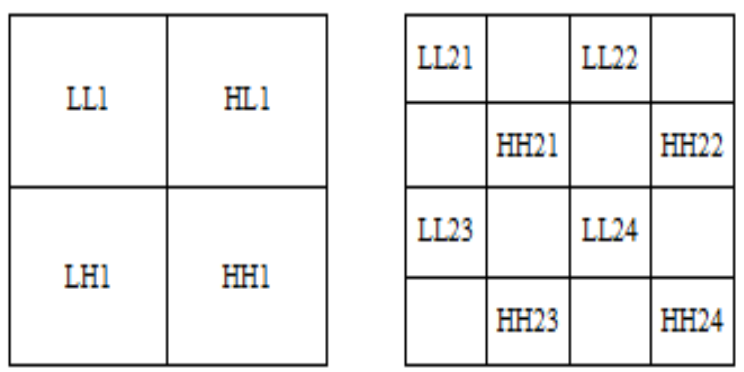

Fig-1: DWT sub-bands in (a) level 1, (b) level 2.

\subsection{Watermark embedding process}

The Watermark embedding process consists of the following steps:

1. Video is divided into frames RGB frames are converted to YUV frames.

2. 2-DWT is applied on it. .

3. RGB watermark image is converted into a vector $\mathrm{P}=\{\mathrm{p} 1, \mathrm{p} 2 \ldots \mathrm{p} 32 \times 32\}$ of zeros and ones.

4. This vector $P$ is again divided into $n$ parts. Then each part is embedded into each of the corresponding LL and $\mathrm{HH}$ sub bands. The watermark pixels are embedded with strength $\mathrm{x}$ into the maximum coefficient Mi of each PC block Yi. The embedding equation is:

$$
\mathrm{Mi}=\mathrm{Mi}+\mathrm{xW}
$$

Where, $\mathrm{x}$ is the watermark embedding strength.

5. Inverse DWT is applied to obtain the watermarked luminance component of the frame. Finally watermarked frame is reconstructed and watermarked video is obtained.

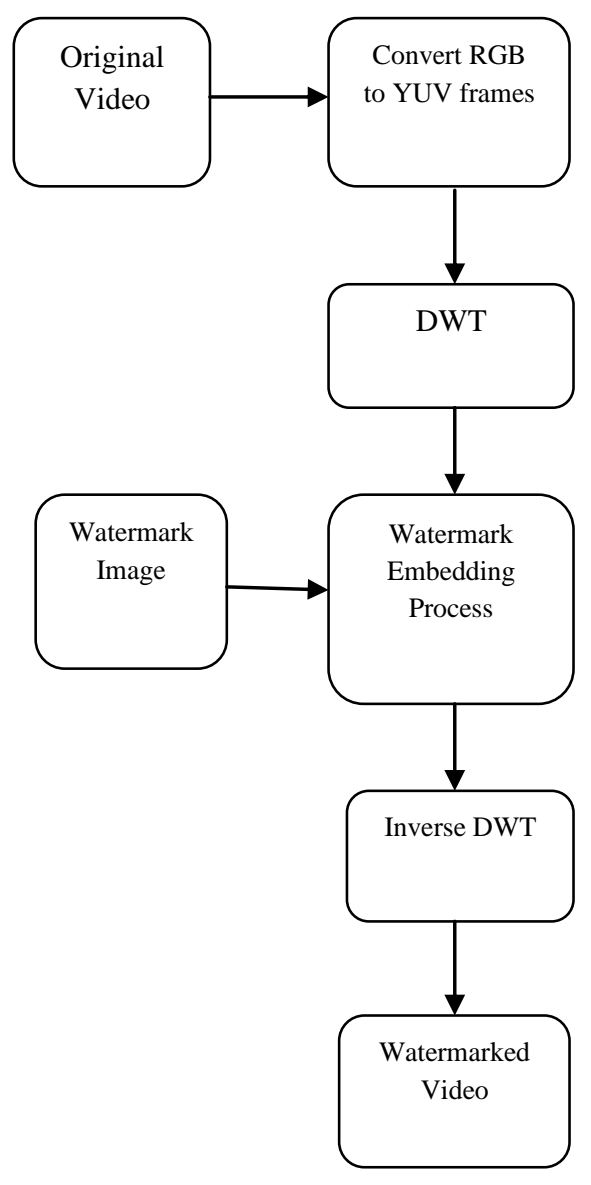

Fig-2: Watermark embedding process.

\subsection{Watermark Extraction Process}

The steps used for watermark extraction is the same as the steps in the embedding but in the reverse direction. As follows

1. Watermarked video is converted into frames. Each RGB frame is converted to YUV representation.

2. DWT is applied. LL and HH sub-bands divided into nxn non-overlapping blocks.

3. Following equation is used to extract watermark

$W=\frac{M_{i}^{1}-M_{i}}{x}$

4. The extracted watermark is compared with the original watermark as follows:

$N C=\frac{\sum_{i} \sum_{j} W(i, j) \cdot W^{\prime}(i, j)}{\sum_{i} \sum_{j} W(i, j)^{2}}(3)$

Where, $\mathrm{NC}$ is the normalized correlation. $\mathrm{NC}$ value is 1 when the watermark and the extracted watermark are identical and zero if the two are different from each other. 


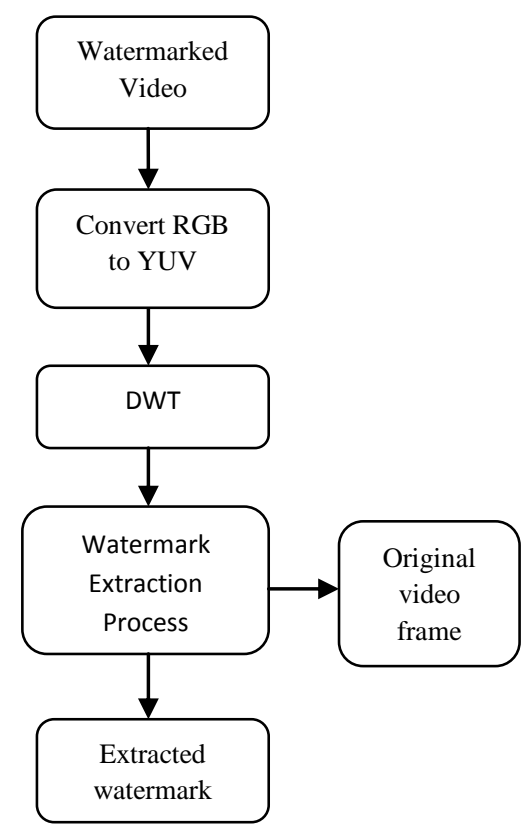

Fig-3: Watermark extraction process.

\section{SIMULINK BASED IMPLEMENTATION}

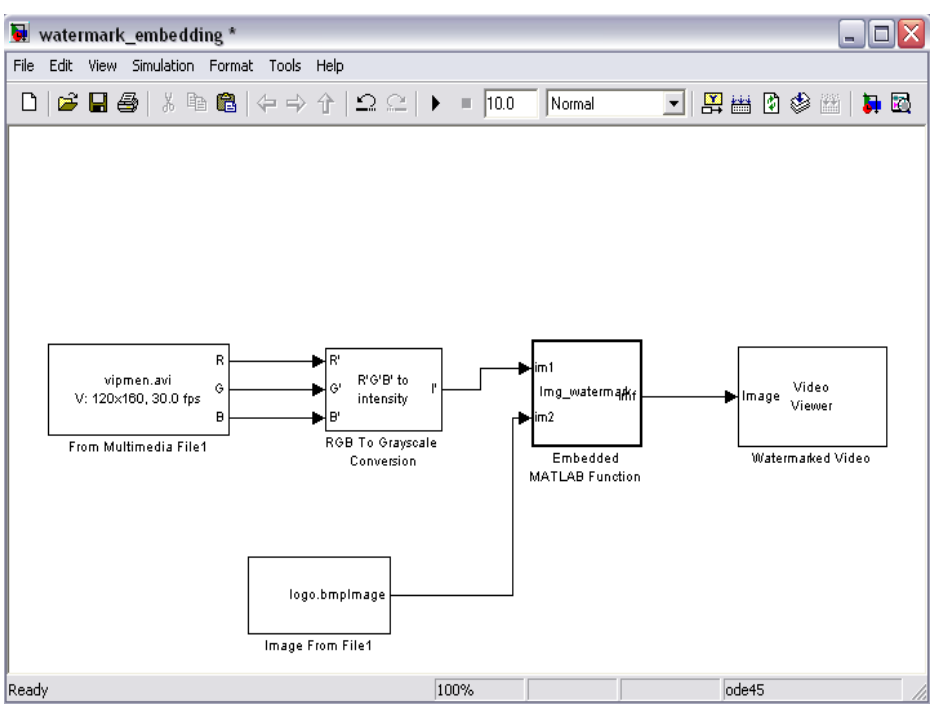

Fig-4: Watermark embedding model in simulink

Simulink is a block diagram environment and Model-Based Design. It supports system-level design, simulation, automatic code creation, and verification of embedded systems. It provides a graphical editor, customizable block libraries for modelling and simulating dynamic systems. It enables to integrate MATLAB algorithms into models and export simulation results to MATLAB for further analysis. Here Matlab-Embedded function block is used to write user define functions such as Watermark Embedding function and extraction function. Resultant simulink models are as shown in fig.

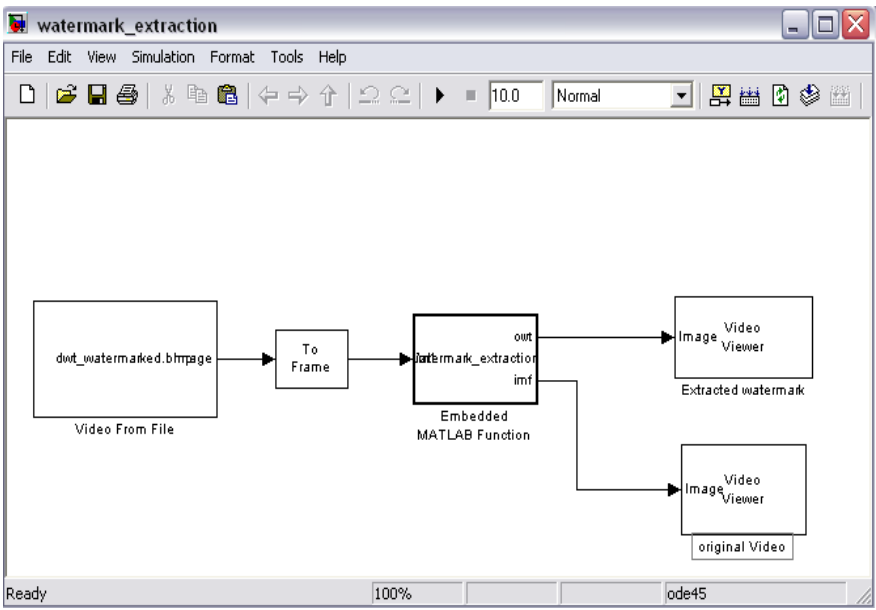

Fig-5: Watermark extraction model in simulink

\section{RESULTS AND DISCUSSION}

Above algorithm is applied to a sample video sequence Vipmen.avi using binary watermark logo. The original sampled frame and its corresponding watermarked frame are shown in Fig. Watermarked frame appears visually identical to the original.

The performance of algorithm can be measured in terms of its imperceptibility and robustness against the possible attacks. Watermarked frame is subjected to a variety of attacks such as Gamma correction, Contrast adjustment,Histogram equalization etc.In case of geometric attacks scheme is tested againstFrame resizing, Frame rotation, Frame cropping.To evaluate the performance of any watermarking system, Peak Signal to Noise Ratio (PSNR) is used as a general measure of the visual quality of the watermarking system.

PSNR: The Peak-Signal-To-Noise Ratio (PSNR) is used to measure deviation of the watermarked and attacked frames from the original video frames and is defined as:

$\mathrm{PSNR}=10 \log _{10}\left(255^{2} / \mathrm{MSE}\right)$

Where MSE (mean squared error ) between the original and distorted frames (size $\mathrm{m} \times \mathrm{n}$ ) is defined as:

$\operatorname{MSE}=(1 / \mathrm{mn}) \sum_{\mathrm{i}=1}^{\mathrm{m}} \sum_{\mathrm{j}=1}^{\mathrm{n}}\left[\mathrm{I}(\mathrm{i}, \mathrm{j})-\mathrm{I}^{\prime}(\mathrm{i}, \mathrm{j})\right]$

Where I and $I^{\prime}$ are the pixel values at location $(i, j)$ of the original and the distorted frame respectively. Higher values of PSNR indicate more imperceptibility of watermarking. It is expressed in decibels $(\mathrm{dB})$.

$\mathrm{NC}$ : The normalized coefficient $(\mathrm{NC})$ gives a measure of the robustness of watermarking and its peak value is 1 . 


$$
\mathrm{NC}=\frac{\sum_{\mathrm{i}} \sum_{\mathrm{j}} \mathrm{W}(\mathrm{i}, \mathrm{j}) \cdot \mathrm{W}^{\prime}(\mathrm{i}, \mathrm{j})}{\sqrt{\sum_{\mathrm{i}} \sum_{\mathrm{j}} \mathrm{W}(\mathrm{i}, \mathrm{j})} \sqrt{\sum_{\mathrm{i}} \sum_{\mathrm{j}} \mathrm{W}^{\prime}(\mathrm{i}, \mathrm{j})}}
$$

Where $\mathrm{W}$ and $\mathrm{W}^{\prime}$ represent the original and extracted watermark respectively.

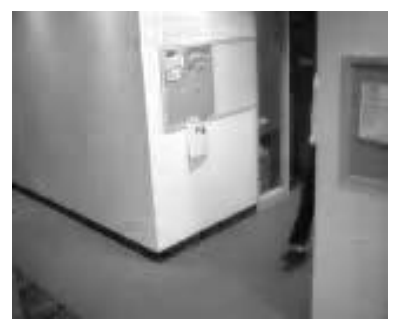

Fig-6: Original Video

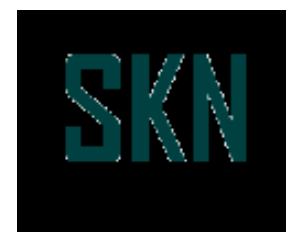

Fig-7: Watermark Image

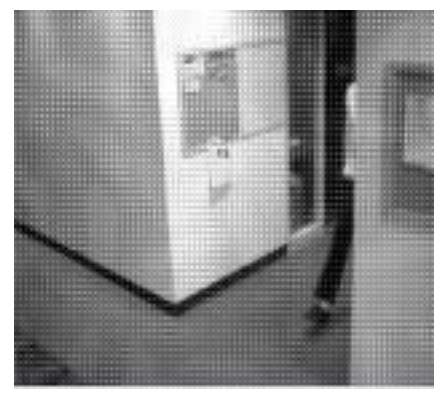

Fig-8: Watermarked Video

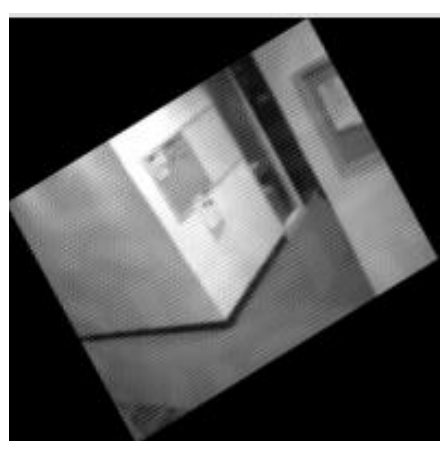

Fig-9: Video frame after rotation by 180 degrees

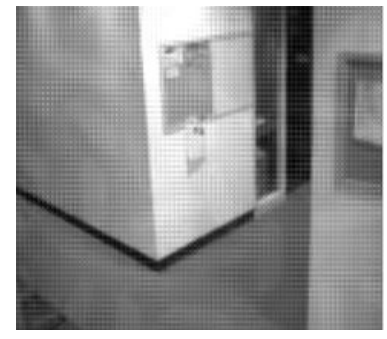

Fig-10: Video frame after resizing

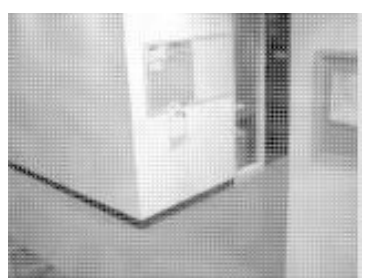

Fig-11: Video frame after gamma correction (Variance 2.2)

Table-1: Values of PSNR and NC when frame is subjected to different attacks.

\begin{tabular}{|l|l|l|}
\hline Attack & PSNR & NC \\
\hline ROTATION & 28.8 & 0.607 \\
\hline RESIZING & 39.46 & 0.6508 \\
\hline $\begin{array}{l}\text { GAMMA } \\
\text { CORRECTION }\end{array}$ & 24.079 & 0.5931 \\
\hline
\end{tabular}

\section{CONCLUSIONS}

Here implementation of digital video watermarking scheme based on DWT is proposed. Due to multiresolution characteristics of DWT this scheme is robust against several attacks. Software model is design by using MATLAB/Simulink. There is no noticeable difference between the watermarked video frames and the original frames. As a futurework this simulink model can be interface with FPGA by using Xilinx system generator block.

\section{REFERENCES}

[1] Nisreen I. Yassin, Nan cy M. Salem, and Mohamed I. El Adawy "Block Based Video Watermarking Scheme Using Wavelet Transform and Principle Component Analysis"IJCSI International Journal of Computer Science Issues, Vol. 9, Issue 1, No 3, January 2012.

[2] HananeH.Mirza, HienD.Thai, Yasunori Nagata and ZenshoNakao" Digital Video Watermarking Based on Principal Component Analysis" in Department of Electrical and Electronics Engineering, University of the Ryukyus Okinawa 9030213,Japan, 2011. 
[3] SanjanaSinha, PrajnatBardhan, SwarnaliPramanick, AnkulJagatramka, Dipak K. Kole, ArunaChakraborty...'Digital Video Watermarking using Discrete Wavelet Transform and Principal Component Analysis"International Journal of Wisdom Based Computing, Vol. 1 (2), August 2011

[4] Snehal V. Patel, Prof. Arvind R. Yadav "Invisible Digital Video Watermarking Using 4-level DWT" National Conference on Recent Trends in Engineering \& Technology, 14 May 2011

[5] KesavanGopal, Dr. M. MadhaviLatha"Watermarking of Digital Video Stream for Source Authentication" IJCSI International Journal of Computer Science Issues, Vol. 7,Issue 4, No 1, July 2010.

[6] Salwa A.K Mostafa, A. S. Tolba, F. M. Abdelkader, Hisham M. Elhindy, "Video Watermarking Scheme Based on Principal Component Analysis and Wavelet Transform" IJCSNS International Journal of Computer Science and Network Security, VOL.9 No.8, August 2009.

[7] Mandeep Singh Saini, VenkataKranthi B, Gursharanjeet Singh Kalra., "Comparative Analysis of Digital Image Watermarking Techniques in Frequency Domain using MATLAB SIMULINK", International Journal of Engineering Research and Applications (IJERA)ISSN: 2248-9622 Vol. 2, Issue 4, May-Jun 2012.

[8] Keshav S Rawat, Dheerendra S Tomar,"Digital watermarking schemes for authorization against copying or piracy of color images" Indian Journal of Computer Science and Engineering Vol. 1 No. 4 295-300

[9] HananeMirza, Hien Thai, and ZenshoNakao,"'Digital Video Watermarking Based on RGB Color Channels and Principal Component Analysis", KES 2008, Part II, LNAI 5178, pp. 125-132, 2008.

[10] Yavuz E., Telatar Z., "Digital Watermarking with PCA Based Reference Images",ACIVS 2007, Springer-Verlag Lecture Notes in Computer Science, 4678, pp.10141023, 2007.

[11] S.Bhargav Kumar, K.Esther Rani,"FPGA Implementation of 4-D DWT and BPS based Digital Image Watermarking"International Journal of Engineering Trends and Technology- Volume3Issue22012.

[12] Saraju P. Mohanty, Renuka Kumara C, and SridharaNayak"FPGA Based Implementation of an Invisible-Robust ImageWatermarking Encoder" CIT 2004, LNCS 3356, pp. 344-353, 2004.

[13] Keshav s rawat "digital watermarking schemes for authorization against copying or piracy of color images" Indian Journal of Computer Science and Engineering Vol. 1 No. 4 295-300.c 